\title{
ELECTRON BEAM LITHOGRAPHY DOUBLE STEP EXPOSURE TECHNIQUE FOR FABRICATION OF MUSHROOM-LIKE PROFILE IN BILAYER RESIST SYSTEM
}

\author{
Kornelia Indykiewicz - Bogdan Paszkiewicz - \\ Tomasz Szymański — Regina Paszkiewicz *
}

\begin{abstract}
The Hi/Lo bilayer resist system exposure in e-beam lithography (EBL) process, intended for mushroom-like profile fabrication, was studied. Different exposure parameters and theirs influence on the resist layers were simulated in CASINO software and the obtained results were compared with the experimental data. The AFM technique was used for the estimation of the e-beam penetration depth in the resist stack. Performed numerical and experimental results allow us to establish the useful ranges of the exposure parameters.

K e y w ords: T-shape resist profile, mushroom-like resist profile, bilayer resist system, resist exposure model, MC
\end{abstract} simulations, e-beam lithography, double step exposure

\section{INTRODUCTION}

The conventional bilayer resist system in configuration $\mathrm{Lo} / \mathrm{Hi}$ is widely used for metal lift-off process in microand nano-technology. This approach provides good quality of uncomplicated spatial geometry metallic structures fabrication with relatively simple process requirements. Metallic structures with complicated 3D geometry, like T-gate or $\Gamma$-gate applied in HEMT transistors, require at least two resist layers with inverse configuration - Hi/Lo, [1-3]. Special preparation and inspection techniques are needed due to the complexity of this approach.

\section{THEORETICAL PART}

Analysis of the electron beam influence on the resist stack is essential part of designing the EBL process, especially for structures with complicated geometry, $[4,5]$. Therefore Monte Carlo simulation, assisted by the CASINO software, was the first step of the research on the T-gate structures fabrication for HEMT transistors.

\subsection{Hi/Lo bilayer resist system}

The well-known resist system for mushroom-shaped profiles, intended for high frequency HEMTs, consists of two materials with different properties. Hi/Lo configuration responds to the sensitivity of the used stack, where the top layer needs a much lower dose-to-clean than the bottom layer, Fig. 1. The energy absorbed in the resist layer strongly affects the solubility of this material during the developing process and as consequence influences the geometry and dimensions of the profile. To avoid unintentional developing of the outer parts of the lightly exposed areas, development was done in two different developers appropriate for the specific resists and having also a weak influence on the other polymer. This development separation, for every layer properly, allows making some assumption for MC simulation. The influence of the electron beam on the Hi/Lo resist stack was conducted in two separated steps.
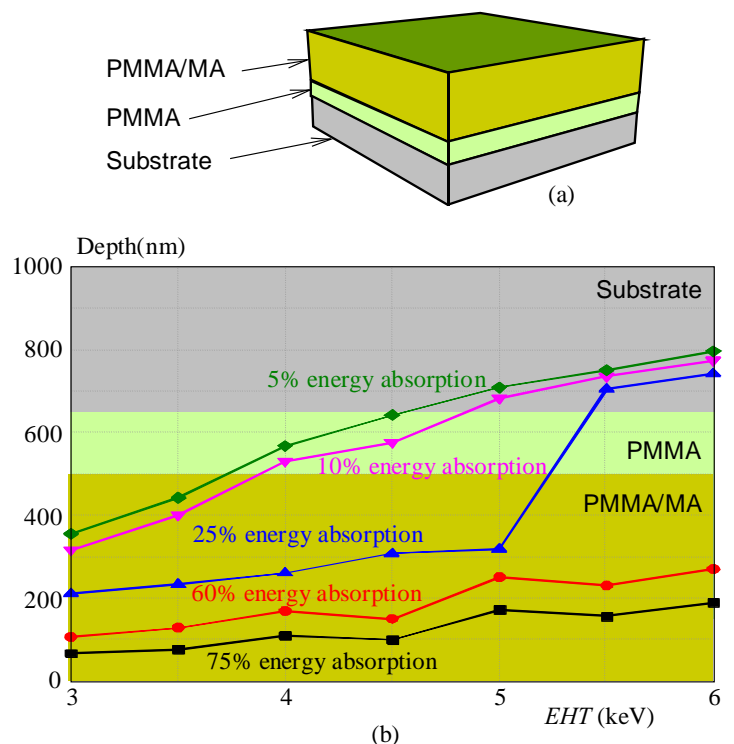

(b)

Fig. 1. (a) - Scheme of used Hi/Lo bilayer resists system, (b) Depth of the percentage energy absorption in the resist stack for different EHT

\footnotetext{
* Faculty of Microsystem Electronics and Photonics, Wroclaw University of Technology, 27 Wybrzeże Wyspiańskiego Str., 50-370 Wrocław, Poland. kornelia.indykiewicz@pwr.wroc.pl
} 

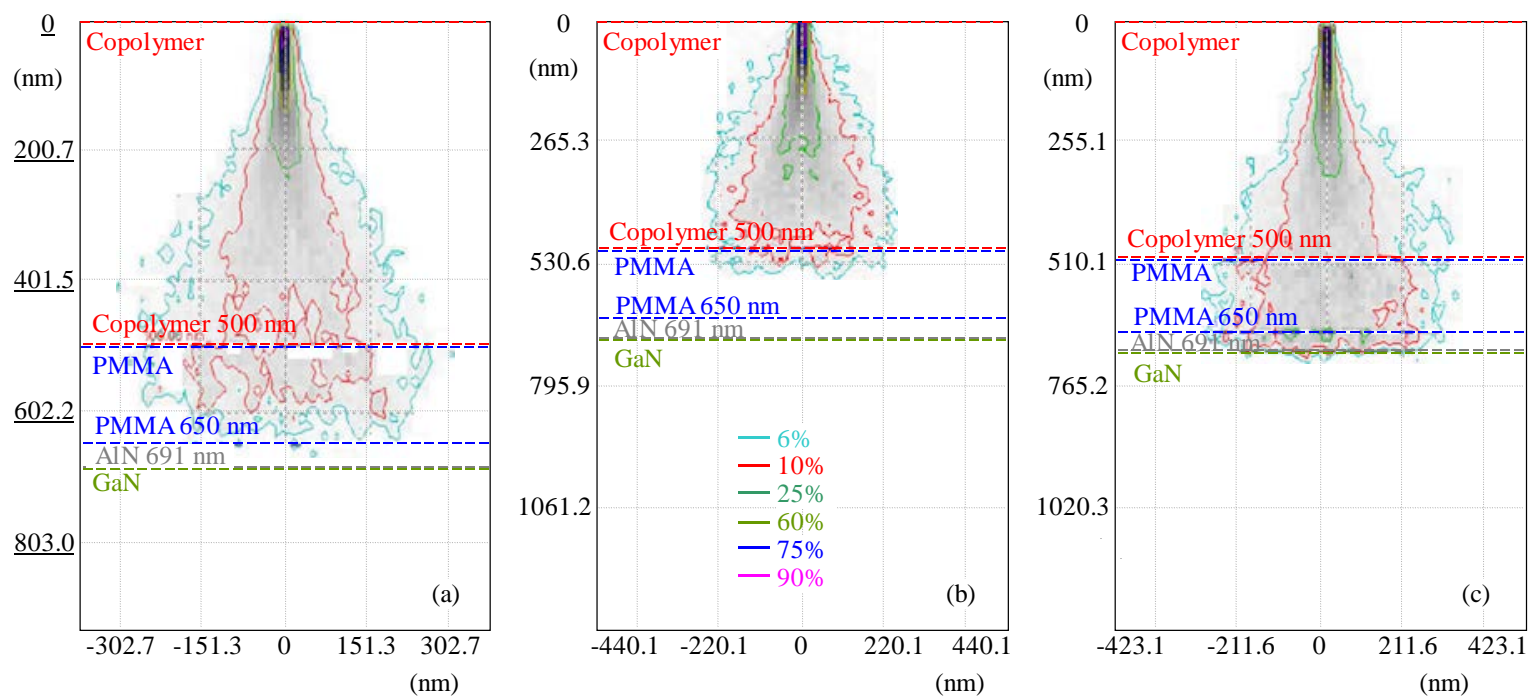

Fig. 2. Distribution of the energy in the resist stack (PMMA/MA + PMMA) for the different acceleration voltage:

(a) $-4.0 \mathrm{kV},(\mathrm{b})-4.5 \mathrm{kV},(\mathrm{c})-5.0 \mathrm{kV}$
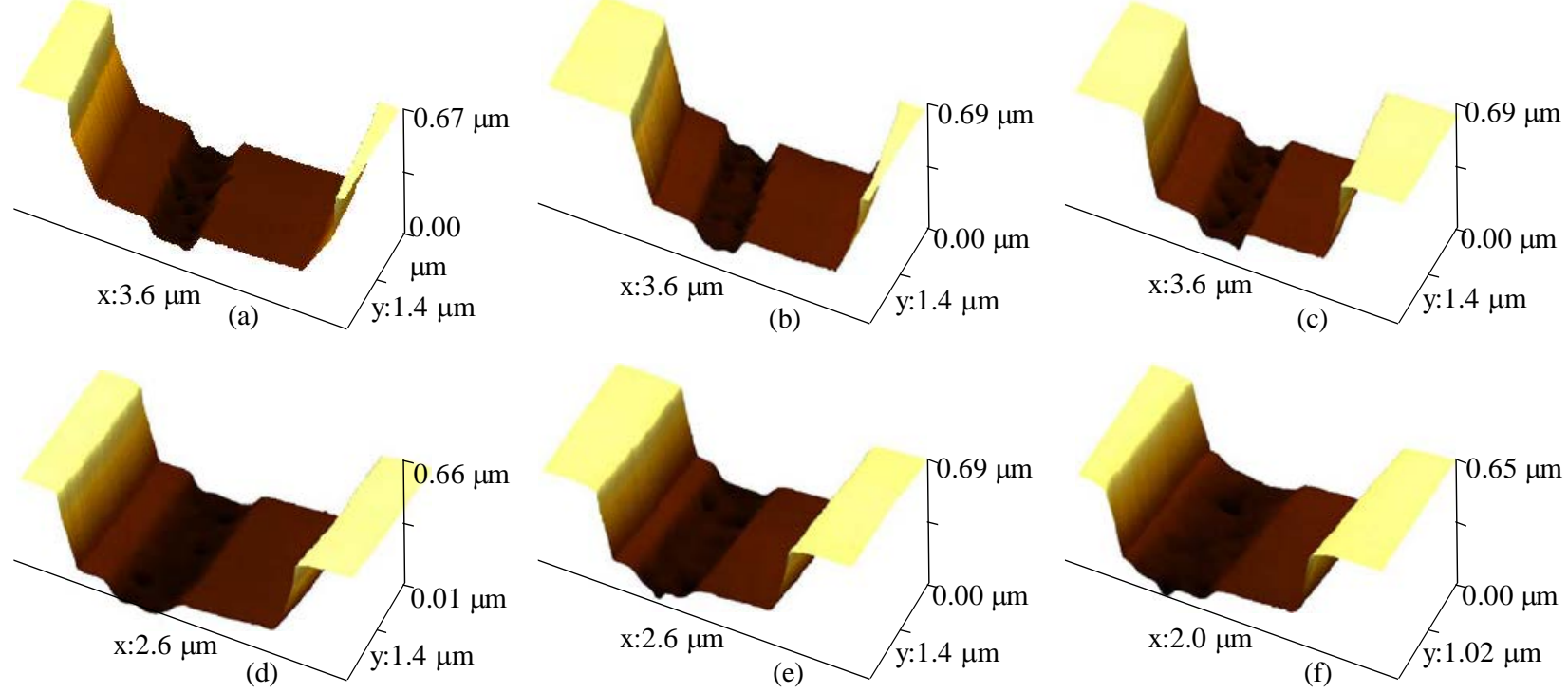

Fig. 3. AFM images for T-shape profiles, designed dimensions: $500 \mathrm{~nm}$ in the bottom resist, and in the top film: (a) $-2000 \mathrm{~nm}$, (b) $1750 \mathrm{~nm},(\mathrm{c})-1500 \mathrm{~nm},(\mathrm{~d})-1200 \mathrm{~nm},(\mathrm{e})-1000 \mathrm{~nm}$, (f) $-750 \mathrm{~nm}$. Applied acceleration voltage for the top resist layer: $5.0 \mathrm{kV}$

\subsection{First step of exposure}

The aim of the first step of exposition was to specify the proper range of the acceleration voltage in which exposure will be performed only in the top resist layer. Simulations were done for 1961 electrons and for 500 $\mathrm{nm}$ of PMMA/MA resist layer $\left(\mathrm{C}_{5} \mathrm{H}_{10} \mathrm{O}_{4}\right)$ and $150 \mathrm{~nm}$ PMMA resist layer $\left(\mathrm{C}_{5} \mathrm{H}_{8} \mathrm{O}_{2}\right)$. The results of simulations for the EHT (Extra High Tension - extremely high electrical voltage) range of $3.0 \mathrm{kV}-6.0 \mathrm{kV}$ are presented in Fig. 1(b). Calculations show the extent of broadening of the pattern dimensions (the proximity effect) due to low EHT application, Fig. 2.

\subsection{Second step of exposure}

The second part of simulations was less demanding. The EHT should be high enough to guarantee a clear window and almost perpendicular shape (light slope) with respect to the substrate. Based on the double step development, simulation was conducted only for PMMA layer.

Analysis of the T-shape profile obtained in Hi/Lo resist stack does not include any model of the process of development based on the absorbed range of energy in the polymer and its influence on the solubility rate. However, authors are aware of the importance of this calculation in designing the process and plan a further research on T-gate fabrication. 

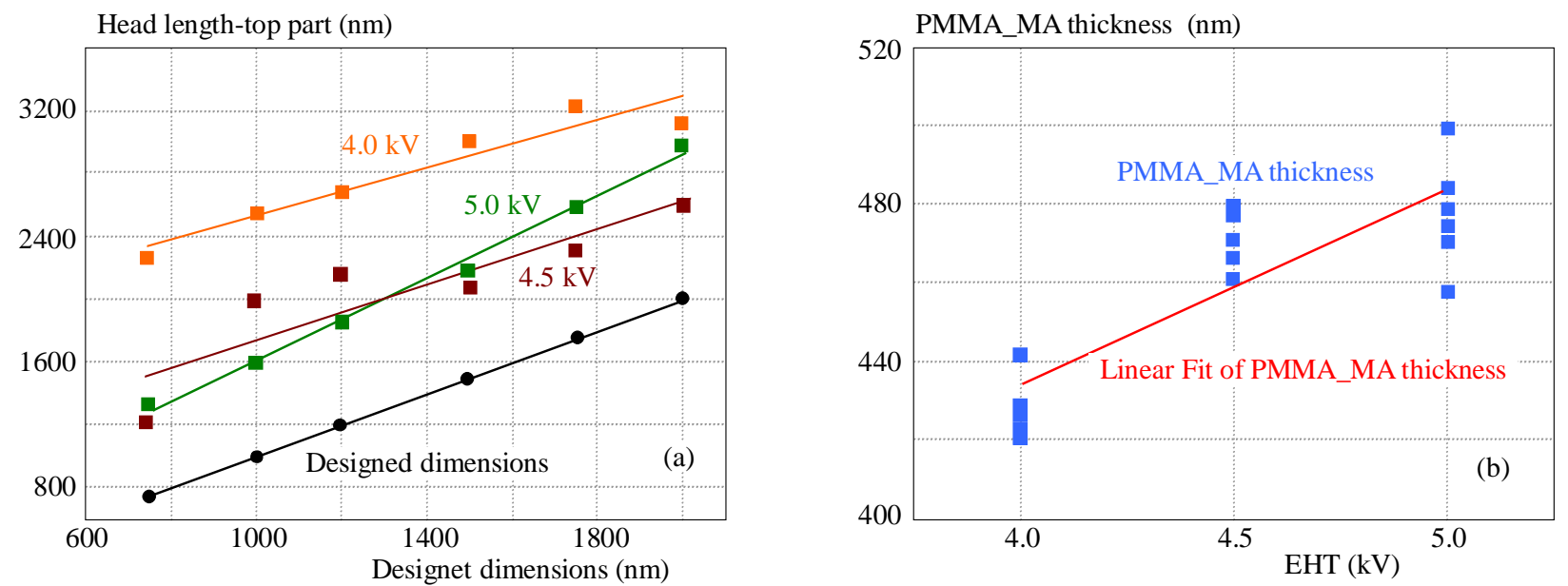

Fig. 4. Results of AFM measurements for T-shape profiles in the resist stack: (a) - length of the top part of the mushroom-like profile ("head") in the PMMA/MA layer for different applied EHT values during the first exposure, (b) - electron beam penetration depth in the PMMA/MA layer for different applied EHT values during the first exposure

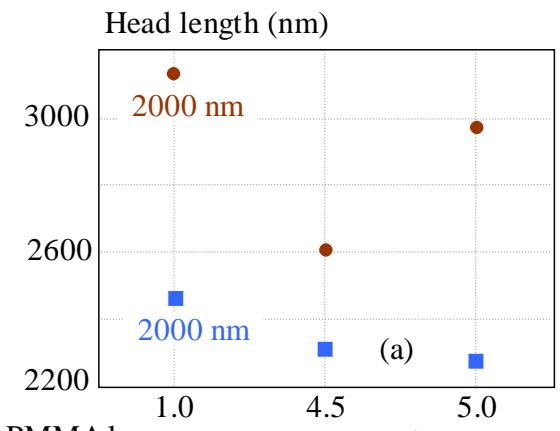

PMMAlayer

Head length (nm)
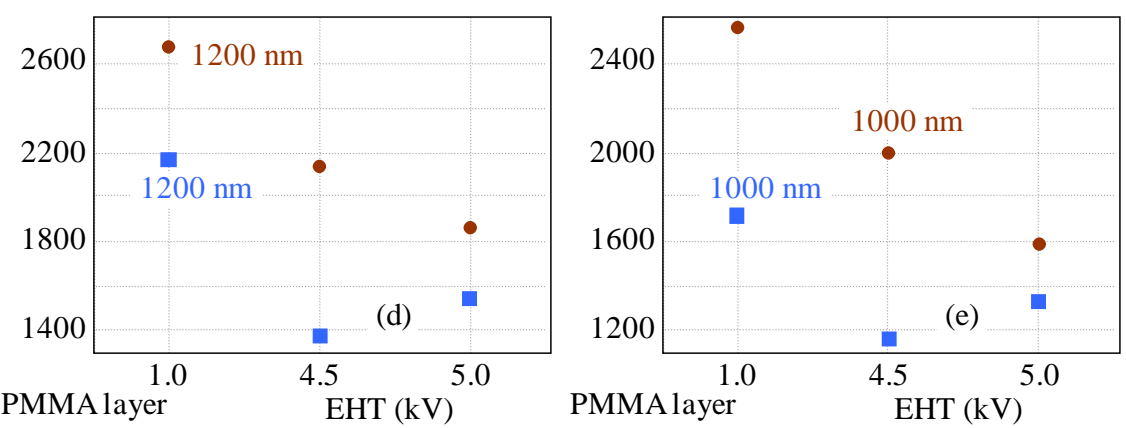

$\begin{array}{ccc}1.0 & 4.5 & \\ \text { PMMAlayer } & \text { EHT }(\mathrm{kV})\end{array}$

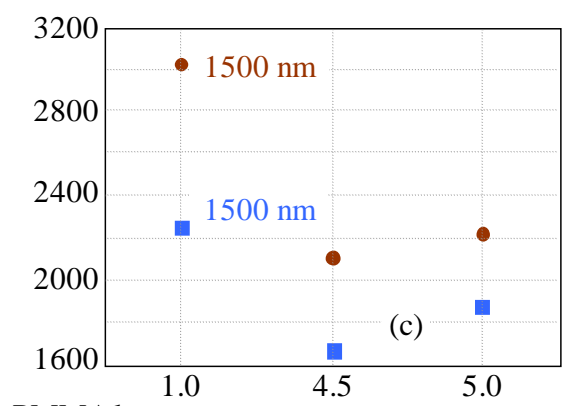

PMMAlayer $\quad$ EHT $(\mathrm{kV})$

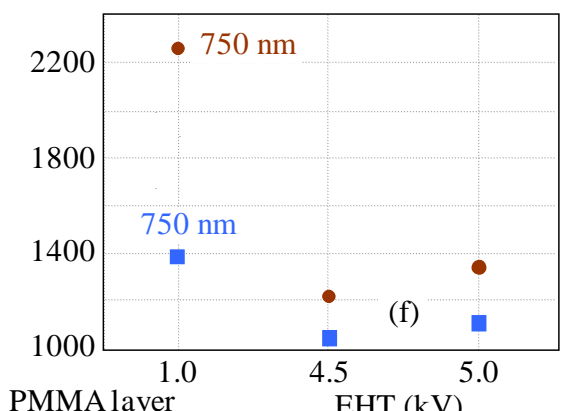

PMMAlayer

Fig. 5. Results of AFM measurements for the "head" part of the T-shape profile in PMMA/MA resist layer for different applied EHT values during the first exposure - circles - top part of the "head", squares — bottom part of the "head". Designed dimensions: (a) $-2000 \mathrm{~nm},($ b) $-1750 \mathrm{~nm},(\mathrm{c})-1500 \mathrm{~nm},(\mathrm{~d})-1200 \mathrm{~nm},(\mathrm{e})-1000 \mathrm{~nm}$, (f) $-750 \mathrm{~nm}$

\section{EXPERIMENTAL PART}

For all conducted experiments a stack of positive resists was used: PMMA $950 \mathrm{k}$ resist layer and PMMA/MA resist layer on AlGaN/AlN/GaN substrates, Fig. 1a. To provide good adhesion of the resist films to the substrate, adhesion primer was used. The thickness of the resulted layers was similar to those applied in the simulated model in CASINO software.

E-beam exposures were conducted in the Raith PIONEER system. The exposure process was divided into two single steps, separated and terminated by the developing stage of processed film. Parameters used in the experiments are shown in Table 1. 

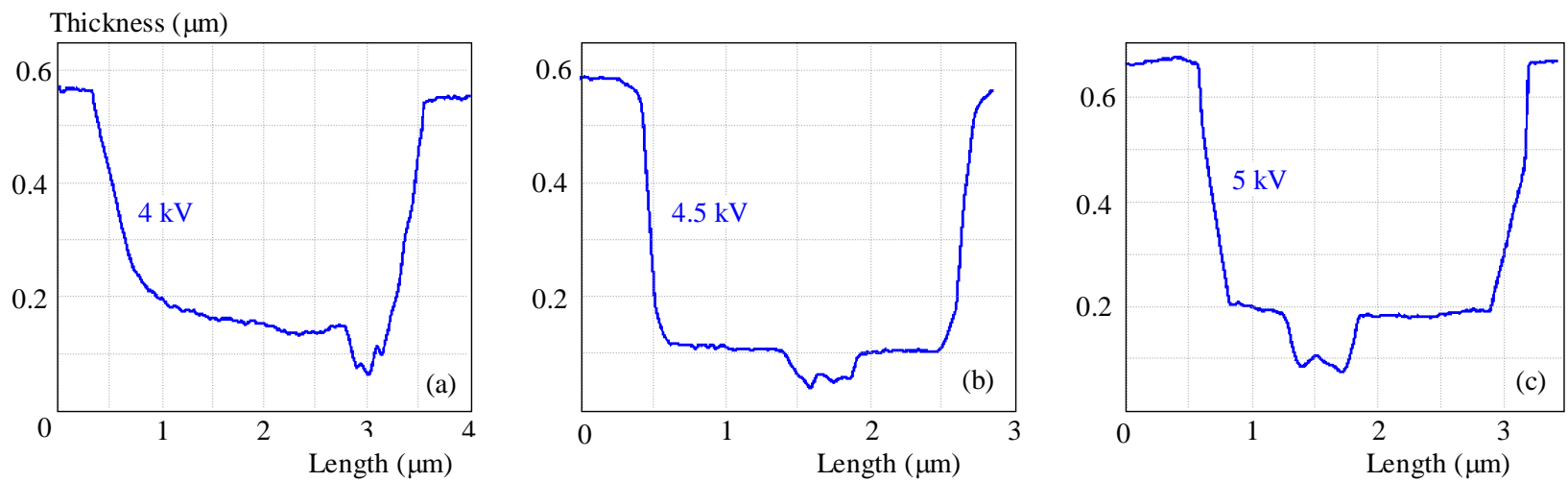

Fig. 6. Results of the AFM measurements of the averaged T-shape profiles in resist stack for different applied EHT values during the first exposure designed dimensions: $1750 \mathrm{~nm} / 500 \mathrm{~nm}$; (a) $-4.0 \mathrm{kV}$, (b) $-4.5 \mathrm{kV}$, (c) $-5.0 \mathrm{kV}$.

Table 1. Double step exposure parameters

\begin{tabular}{ccccc}
\hline Process & Layer & $\begin{array}{c}\text { RHT } \\
(\mathrm{kV})\end{array}$ & $\begin{array}{c}\text { Area Dose } \\
\left(\mu \mathrm{C} / \mathrm{cm}^{2}\right)\end{array}$ & Developer \\
\hline I step & PMMA/MA & $4.0 ; 4.5 ; 5.0$ & 25 & AR 600-50 \\
II step & PMMA & 25 & 100 & AR 600-56 \\
\hline
\end{tabular}

The aim of the first exposure was to create the top part of the profile — "head" — in the PMMA/MA layer without influencing the bottom resist layer. According to the simulation results, EHT was used in the range of: $4.0 \mathrm{kV}$ to $5.0 \mathrm{kV}$.

The second exposure provides the formation of the bottom part of the profile - "leg" — in the PMMA layer. Exposure should takes place in only the PMMA layer.

T-shape profile geometry structures were designed with dimensions as follow: bottom part of the profile constant width for all structures - $500 \mathrm{~nm}$; top part of the profile — in the range from $750 \mathrm{~nm}$ to $2000 \mathrm{~nm}$ with $250 \mathrm{~nm}$ steps.

\section{DISCUSSION}

Fabricated structures were examined by AFM microscope using high aspect ratio AFM tips. The measured AFM images were obtained by surface reconstruction. Fabricated T-shape profiles with designed dimensions $750 \mathrm{~nm}$ to $2000 \mathrm{~nm} / 500 \mathrm{~nm}$ and $\mathrm{EHT}=5.0 \mathrm{kV}$ for the first exposure are presented in Fig. 3.

Big discrepancies were observed between the design and created geometry dimensions of the fabricated structures in the resist stack for all conducted EHT values, Fig. 4(a). Black circles present the designed dimensions of the "head" part of the profile. The coloured points present the measured length of the fabricated "head" just under the PMMA/MA surface. For the lowest EHT values - blue squares - the dimensions are much wider than were planned. Application of higher EHT values results in smaller disproportion between the design and experiments. The observed effects are a consequence of the nature of the e-beam impact in the solid - the measurements confirm our assumption and show the extent of this effect in the resist stack.

AFM measurements of the PMMA/MA layer thickness after the first step of process show, according to the simulation results, that the increase in EHT during the first exposure strongly influences the e-beam penetration depth, Fig. 4(b). This confirms the correctness of the initial process assumptions.

There is also a big difference in the dimensions on each profile in PMMA/MA layer between the bottom and top part of the "head" of the profile, Fig. 5.

Analysis of the profiles for different EHT values applied in the first exposures suggested also application of higher acceleration voltages, due to the incorrect profiles geometry, Fig. 6. Low EHT values negatively influence the bottom profile - the first window is not truly "clean" and acts as a mask during the second step of development. The profile of the "leg" is not properly defined, especially for $4.0 \mathrm{kV}$, Fig. $6(\mathrm{a})$.

\section{CONCLUSIONS}

Preliminary studies were presented of T-shape profile fabrication in a bilayer resist system intended for HEMT metallic mushroom gate lift-off process. Numerical analysis of the influence of the electron beam on the Hi/Lo resist stack in a double step exposure method was conducted and compared with experiments. The obtained results showed the importance of MC simulation of the exposure process of EBL and need to be complemented by analyses of the development model and simulations.

For further verification, the fabricated structures will be measured by SEM. The cross-section of the fabricated profiles will be examined, especially to confirm the profile geometry due to the limitation of the AFM technique. This will allow verification of the usefulness of the structures for metallic T-gate HEMT lift-off process. 


\section{Acknowledgement}

This work was co-financed by the European Union within European Regional Development Fund, through grant Innovative Economy (POIG.01.01.02-00-008/0805), National Centre for Science under the grant no. N N515 495740, by National Centre for Research and Development through Applied Research Program grant no. 178782, by Wroclaw University of Technology statutory grants and Slovak-Polish International Cooperation Program no. SK-PL-0005-12.

\section{REFERENCES}

[1] ROBIN, F.-MEIER, H.-HOMAN, O. J.-BACHTOLD, W. : A novel Asymmetric gate recess process for InP HEMTs, 14th Indium Phosphide and Related Materials Conference, 2002, pp. 221-224.

[2] CHANG, E. Y.-LIN, K. C.-LIU, E. H.-CHANG, C. Y.CHEN, T. H.-CHEN, J. : Submicron T-Shaped Gate HEMT Fabrication Using Deep-UV Lithography, IEEE Electron Device Letters 15 No. 8, 277-279.

[3] TODOKORO, Y.: Double-Layer Resist Films for Submicrometer Electron-Beam Lithography, IEEE Transactions on Electron Devices 27 No. 8, 1443-1448.

[4] HERZOG, R. F.-GREENEICH, J. S.-EVERHART, T. E.: Computer-Controlled Resist Exposure in the Scanning Electron Microscope, IEEE Transactions on Electron Devices 19 No. 5, 635-641.

[5] GREENEICH, J. S. :VAN DUZER, T.: Model for Exposure of Electron-Sensitive Resists, Journal of Vacuum Science and Technology 10 No. 6, 1056-1059.
Received 15 June 2014

Kornelia Indykiewicz (MSc, Eng), born in Wyrzysk, Poland in 1986, is a $\mathrm{PhD}$ student at Wroclaw University of Technology, Department of Microelectronics and Nanotechnology. She works in a multidisciplinary team doing research concerning AlGaN/GaN based transistors.

Bogdan Paszkiewicz received his MSc degree in Electrical Engineering from St. Petersburg Electrotechnical University, St. Petersburg, Russia in 1979 and PhD degree from the Wroclaw University of Technology in 1997. Now he is assistant professor at WUT. His research is focused on the design and parameter evaluation of nitrides-based devices: HEMTs and sensors. He is co-author of 211 scientific publications. His articles were cited 155 times.

Tomasz Szymanski (MSc, Eng) born in Legnica, Poland in 1988 is the second year PhD student at Wroclaw University of Technology, Department of Microelectronics and Nanotechnology. He works in a multidisciplinary team doing research concerning AlGaN/GaN based transistors and epitaxial structures.

Regina Paszkiewicz received her MSc degree in Electrical Engineering from St. Petersburg Electrotechnical University, St. Petersburg, Russia in 1982 and $\mathrm{PhD}$ degree from the Wroclaw University of Technology in 1997. Now she is full professor at WUT. Her research is focused on the technology of ( $\mathrm{Ga}, \mathrm{Al}, \mathrm{In}) \mathrm{N}$ semiconductors, microwave and optoelectronic devices technological processes development. She is co-author of 225 scientific publications. Her articles were cited 230 times.

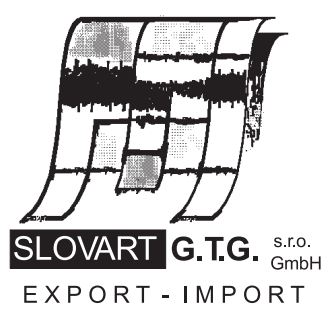

EXPORT - IMPORT
E X P OR T - I M P OR T

of periodicals and of non-periodically printed matters, books and CD-ROMs

Krupinská 4 PO BOX 152, 85299 Bratislava 5, Slovakia tel: ++421 263839 472-3, fax: ++421263839485 info@slovart-gtg.sk; http://www.slovart-gtg.sk

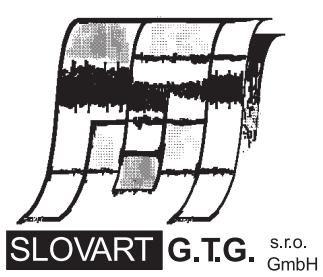

EXPORT - IMPORT 